\title{
Valorisation de poudrettes de pneus par incorporation dans du PE recyclé Influence de la morphologie et des traitements de surface des poudrettes sur le comportement mécanique du matériau
}

\author{
SONNIER Rodolphea, CLERC Laurenta, LEROY Erica, \\ LOPEZ-CUESTA José-Mariea, BERGERET Anne \\ CMGD, Ecole des Mines d'Alès \\ 6, avenue de Clavières, 30319 ALES cedex
}

laurent.clerc@ema.fr

\begin{abstract}
RÉSUMÉ
L'influence de l'incorporation de poudrettes de pneumatiques commerciales dans du polyéthylène (haute et basse densité) recyclé sur les propriétés mécaniques de mélanges PE/poudre a été étudiée. Les résultats des tests mettent en évidence la chute des performances en raison de l'absence d'adhésion matrice/particule. Deux traitements de compatibilisation ont donc été expérimentés. L'oxydation par irradiation gamma de la poudrette et son incorporation dans une matrice contenant une petite quantité de fonctions anhydride maléique n'a pas permis d'améliorer les propriétés mécaniques, bien que l'oxydation ait été effective. Un traitement par permanganate de potassium a donné au contraire des résultats intéressants, mais le mécanisme expliquant l'augmentation du module d'Young n'est pas encore élucidé.
\end{abstract}

\begin{abstract}
In this study, polymer compounds consisting in a recycled polyolefin matrix (high or low density polyethylene (HDPE or LDPE)) and ground tyre rubber (GTR) powder are developed.The major problem in this two phase systems is the very poor interfacial adhesion between thermoplastic matrix and rubber filler, which impairs the mechanical properties of the compound. Two treatments have been tested to improve the adhesion and so the mechanical performances: GTR powder was oxidized either by reaction with potassium permanganate aqueous solution (0-20\%wt of $\mathrm{KMnO}_{4}$ ) or by irradiation (gamma-irradiation : 0-100 kGy). Oxydized GTR powders were then introduced into a HDPE matrix containing Maleic Anhydride grafted HDPE as a compatibilizing agent. While no significant improvement of mechanical properties was observed for the irradiated GTR powder, the $\mathrm{KMnO} 4$ treatment lead to improved Young's modulus and yield stress, but the adhesion mechanism involved was not clearly identified.
\end{abstract}

\section{INTRODUCTION}

Le développement durable est désormais un objectif proclamé au niveau international et la gestion des déchets doit être de plus en plus prise en compte par les industriels. Dans ce cadre, la fin de vie des pneus pose un véritable problème écologique. Aux Etats-Unis, par exemple, il y a plus de 250 millions de pneus usagés par an. En Europe, une directive interdit désormais le stockage de ces déchets. Dans ces conditions, le recyclage des pneus exige de nouveaux débouchés qui peuvent devenir rentables. L'un de ces débouchés est le broyage du pneu en poudrette et l'incorporation de celle-ci dans un matériau thermoplastique. Mais la compatibilité entre la matrice thermoplastique et la poudre de pneus est faible et les propriétés mécaniques de tels mélanges sont insuffisantes pour des applications de haute performance [I]. Diverses solutions sont envisageables pour améliorer ces propriétés :

- le contrôle de la morphologie des particules (taille et rugosité) en est une, mais cette possibilité s'avère insuffisante dans de nombreux cas [2-3],

- la dévulcanisation du caoutchouc [2-4],

- l'application de traitements de surface des particules afin de compatibiliser la matrice et la poudrette. Ainsi, le caoutchouc peut être fonctionnalisé par des traitements physiques [5-7] ou chimiques [8-9] qui génèrent en surface des groupes polaires ou permettent de greffer des molécules,

- une revulcanisation durant le mélange peut constituer une autre voie pour créer des liaisons et améliorer l'adhésion interfaciale [10].

Dans ce travail, nous nous sommes intéressés à l'étude de mélanges PE recyclé / poudre de pneus. Nous avons tout d'abord étudié l'influence des caractéristiques de la poudre (taux de poudre incorporée, diamètre moyen des particules et présence de fibres résiduelles) sur les propriétés mécaniques des mélanges obtenus. Dans un second temps, nous avons étudié l'effet, sur les performances mécaniques des mélanges obtenus, de deux traitements de surface différents : l'un physique (oxydation par irradiation gamma) et l'autre chimique (oxydation 
par permanganate de potassium, traitement proposé par Liu [9]).

\section{MATÉRIAUX ETTECHNIQUES UTILISÉES}

\section{Matériaux}

Les polyéthylènes haute et basse densité sont des matériaux recyclés contenant une petite quantité de polypropylène. Le taux de cristallinité est de 48 \% pour le PEhd et 30 \% pour le PEbd. Le polyéthylène greffé anhydride maléique est de marque OREVAC I 8302 d'ATOFINA. || contient 0,5\% en poids de fonctions anhydride maléique.

Les poudrettes de pneus proviennent de différents sites de production d'Europe et sont nommées dans cette étude G,T5, T5PL, T6,T8, R8 et E3. Elles ont toutes été obtenues par broyage à température ambiante. Le tableau I regroupe des informations concernant ces poudres.

Le permanganate de potassium (99\%) a été fourni parACROS ORGANICS.

\begin{tabular}{|c|c|c|c|c|}
\hline Poudre & $\begin{array}{c}\text { Origine (poids lourds : PL ; } \\
\text { voiture : VL) }\end{array}$ & $\begin{array}{c}\text { Présence de } \\
\text { fibres }\end{array}$ & $\begin{array}{c}\text { Diamètre moyen } \\
\text { des particules } \\
(\boldsymbol{\mu m})\end{array}$ & $\begin{array}{c}\text { Diamètre } \\
\text { médian }(\boldsymbol{\mu m})\end{array}$ \\
\hline G & VL + PL & Nombreuses & 383 & 374 \\
\hline T5 & VL+PL & Rares & 515 & 510 \\
\hline T5PL & PL & Non & 503 & 490 \\
\hline T6 & VL+PL & Rares & 623 & 613 \\
\hline T8 & VL + PL & Rares & 692 & 682 \\
\hline R8 & VL + PL & Rares & 709 & 689 \\
\hline E3 & VL + PL & Rares & 1217 & 1186 \\
\hline
\end{tabular}

Tableau I - Caractéristiques des poudrettes utilisées.

\section{Mise en œuvre et analyse}

Les mélanges ont été réalisés à l'aide du mélangeur interne de marque Haake. Le polyéthylène est introduit dans la cuve à $130^{\circ} \mathrm{C}$ (PEbd) ou $145^{\circ} \mathrm{C}$ (PEhd), avec le PEgMA le cas échéant. La vitesse des rotors est de $60 \mathrm{rpm}$. Lorsque le polyéthylène est fondu (stabilisation du couple), après 4 minutes environ, la poudre est introduite. Le mélange se poursuit pendant encore 6 minutes. Les éprouvettes normalisées sont obtenues par moulage par injection à $170^{\circ} \mathrm{C}$ (presse Sandretto).

Les essais mécaniques ont été réalisés avec les presses ADAMEL MTS (flexion) et ZWICK ZOIO (traction) selon les normes ISO 178 : I 993 et ISO 527- I : 1993. L'essai de flexion est réalisé à une vitesse de $2 \mathrm{~mm} / \mathrm{min}$ tandis que l'essai de traction est effectué à $100 \mathrm{~mm} / \mathrm{min}$. 10 éprouvettes sont testées pour chaque essai et chaque mélange.

La mouillabilité des poudres a été suivie à l'aide du mouillabilimètre LPR 902 de KSV Instruments Ltd. Un solvant (ici, l'éthanol) est absorbé par un lit de poudre. Un laser traverse le liquide et un détecteur mesure la variation d'intensité et donc le volume de solvant absorbé par la poudre. Le carré de ce volume est tracé en fonction du temps. La pente $A$ de la courbe, représentative de la vitesse de montée capillaire du liquide au travers du lit de poudre, est égale à :

$$
A=\eta /\left(C^{*} \gamma_{L}^{*} \cos \theta\right)
$$

Avec:

$\eta$ : la viscosité du liquide,

C: une constante du matériau indépendante du liquide utilisée

(C dépend de la porosité et du tassement des particules),

$\gamma_{L}$ : l'énergie libre de surface du liquide,

$\theta$ : l'angle de contact

Ainsi l'évolution de la pente est une mesure de l'affinité entre la poudre et le solvant.

Les observations microscopiques ont été effectuées à l'aide d'un microscope électronique à balayage de marque Jeol. Les conditions d'observations sont indiquées sur les photographies.

Les échantillons ont été également analysés par spectroscopie infrarouge à l'aide de l'appareil de marque PerkinElmer en mode ATR. Le cristal utilisé est ZnSe (orientation 45). Le spectre final est une moyenne de 10 analyses.

\section{INFLUENCE DES CARACTÉRISTIQUES DE LA POUDRE}

\section{Influence du taux de charge}

L'incorporation de poudre dans la matrice polyéthylène fait chuter les propriétés mécaniques du compound comme le montrent les figures 2 et 3. La diminution du module d'Young, de la contrainte max et de l'allongement à la rupture est proportionnelle au taux de charge. Pour des taux élevés, l'allongement à la rupture se stabilise, dû probablement à un phénomène de percolation.

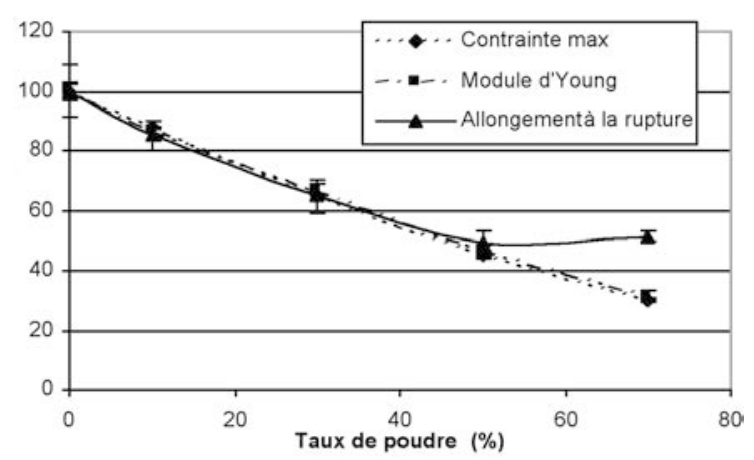

Figure 2 - Evolution des propriétés mécaniques de mélanges PEbd/poudre $G$ en fonction du taux de charge (en \% de la valeur du PEbd vierge). 


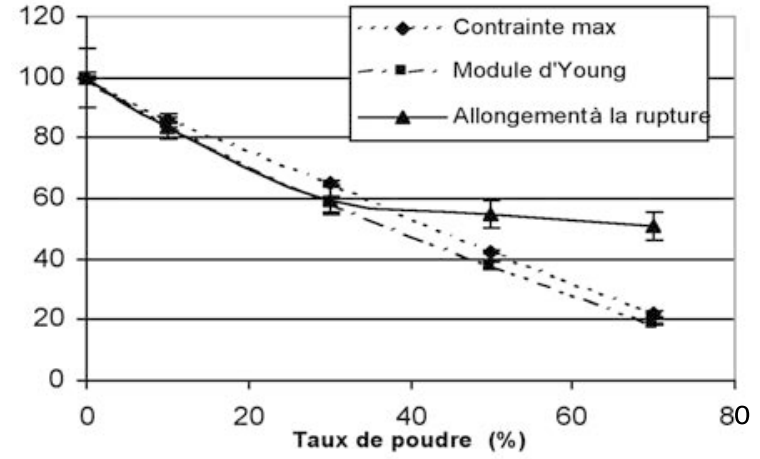

Figure 3 - Evolution des propriétés mécaniques de mélanges PEhd/poudre $G$ en fonction du taux de charge (en \% de la valeur du PEhd vierge).

Comme on peut le voir sur la figure 4 représentant le faciès de fracture d'un mélange PEbd/poudre 50/50, la chute drastique des propriétés mécaniques peut être expliquée par l'absence d'adhésion interfaciale entre les particules et la matrice.

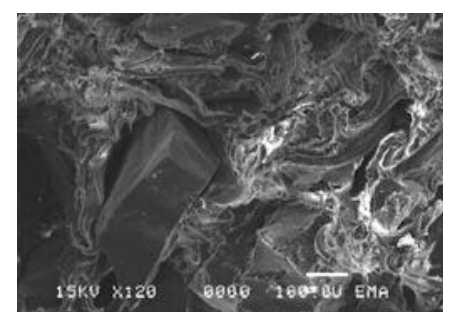

Figure 4 - Osservation d'un faciès de fracture d'un mélange PEbd/poudre G50/50.

\section{Influence de la granulométrie}

Différentes poudres, toutes obtenues par un broyage à température ambiante, mais de provenance et surtout de granulométries différentes, ont été incorporées dans du polyéthylène basse densité (taux de charge $50 \%$ ). La figure 5 présente les résultats des essais mécaniques réalisés. Nous n'observons aucune corrélation entre le diamètre moyen des particules et le module de flexion (ou la contrainte max). Cependant, l'allongement à la rupture semble diminuer avec une augmentation du diamètre moyen.

Ces résultats montrent que la granulométrie de la poudre a peu d'influence sur les performances mécaniques. Le contrôle de la granulométrie ne permet pas d'améliorer sensiblement le comportement mécanique des mélanges PE/poudre.
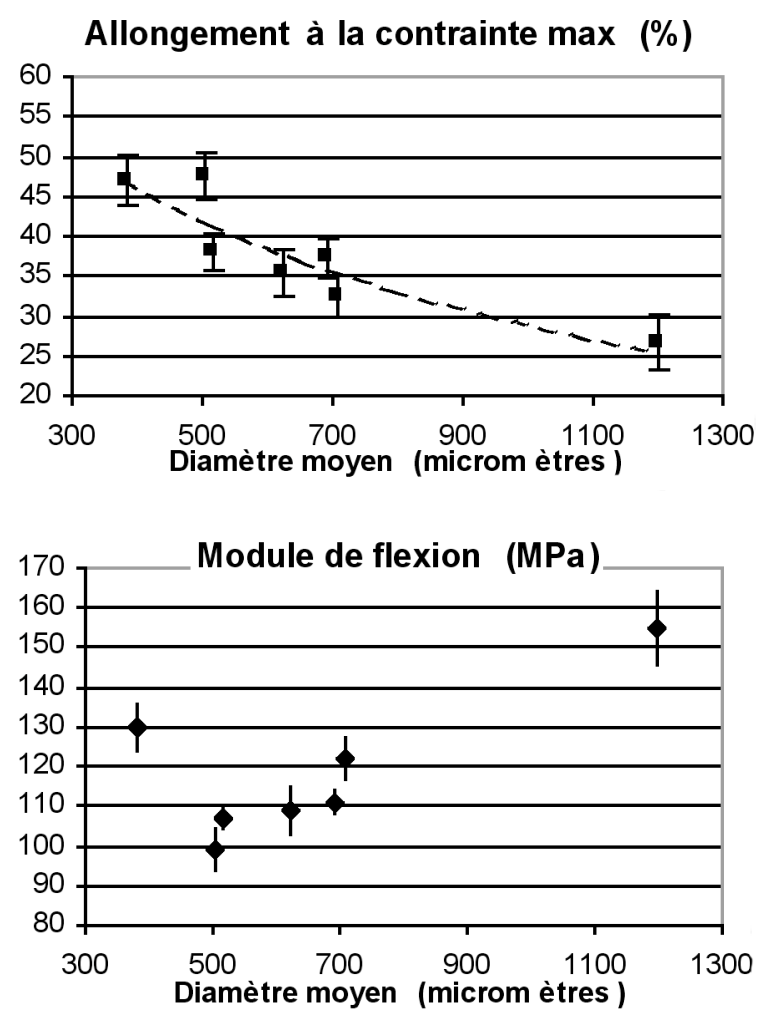

Figures 5 - Propriétés mécaniques de mélanges PEbd/poudre 50/50 pour différentes poudres.

\section{Influence du taux de fibres}

La poudre T5PL, dépourvue initialement de fibres, a été mélangée avec des fibres PET issues du procédé de broyage des pneus dans des proportions connues. Le mélange a été introduit dans du PEbd (50 \% de PEbd). La figure 6 illustre l'influence du taux de fibres sur les propriétés mécaniques : le module d'Young et la contrainte max augmentent sensiblement tandis que l'allongement à la rupture diminue lorsque le taux de fibres augmente. Cela indique que, lors du broyage, il peut être intéressant de ne pas éliminer totalement les fibres et d'obtenir ainsi une poudre contenant des fibres en quantité non négligeable afin de renforcer la matrice polyéthylène.

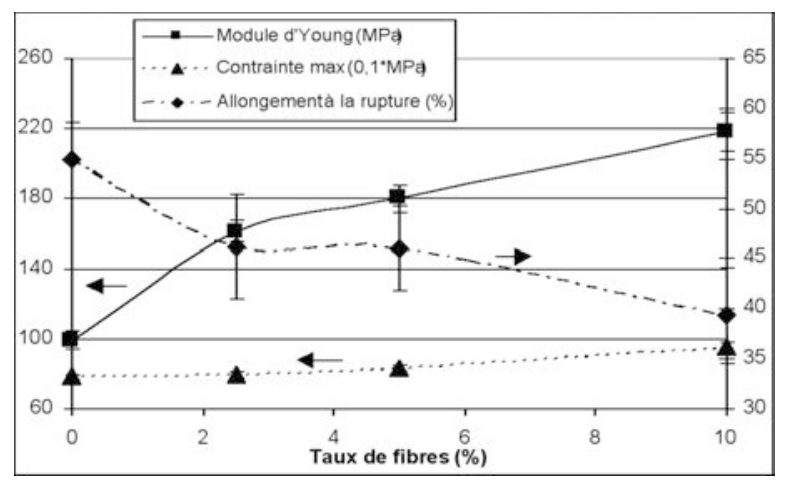

Figure 6 - Propriétés mécaniques de mélanges PEbd/poudre T5PL/fibres en fonction du taux de fibres (50\% de polyéthylène). 


\section{Amélioration des propriétés par tamisage}

Pour confirmer les résultats obtenus précédemment, nous avons réalisé une séparation granulométrique sur la poudre $G$. La fraction inférieure à 104 um a été incorporée dans du PEbd (taux de charge $50 \%$ ). Les propriétés mécaniques de ce mélange ainsi que du PEbd pur et d'un mélange PEbd/G (non tamisée) 50/50 sont représentées sur la figure 7. La contrainte max et surtout le module de flexion sont très nettement améliorés avec la poudre tamisée, comparativement au mélange avec la poudre initiale. Le module est même de l'ordre de celui du PEbd vierge. Cela n'est pas dû à la diminution de la granulométrie mais à l'enrichissement de la poudre en fibres courtes, comme nous pouvons l'observer sur la figure 8. La fraction fine est enrichie en fibres.

Cependant, pour les produits étudiés, cette fraction représente moins de $10 \%$ de la masse de poudre. Aussi est-il nécessaire d'explorer d'autres voies pour pouvoir recycler des quantités importantes de pneus. La modification de surface des particules de caoutchouc est une voie possible.

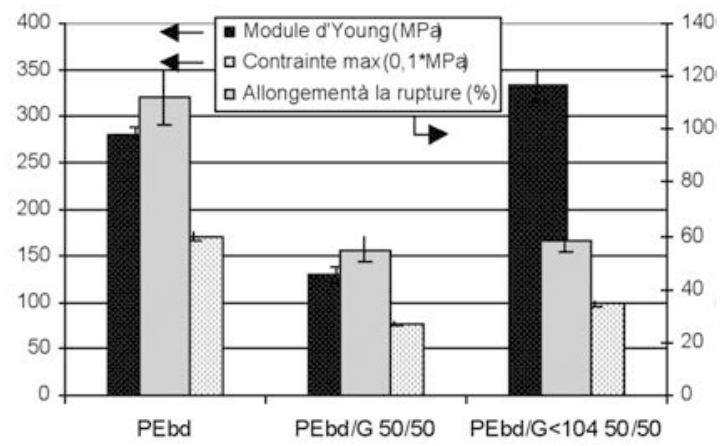

Figure 7 - Propriétés mécaniques des mélanges PEbd, PEbd/G 50/50 et PEbd/G<104 50/50.

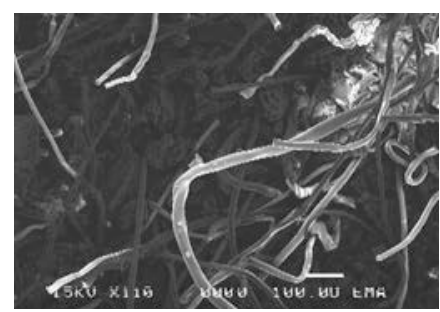

Figure 8 - Ooservation (MEB) de la poudre $G<104 \mu \mathrm{m}$.

\section{TRAITEMENTS DE SURFACE}

La modification de surface consiste à créer des groupes fonctionnels à la surface des particules afin d'améliorer la compatibilisation de celles-ci avec la matrice. Dans notre cas, il est également nécessaire de créer des groupes fonctionnels dans la matrice. En effet, le polyéthylène est apolaire et ne présente pas de tels groupes. Aussi avons-nous incorporé dans le mélange $5 \%$ de polyéthylène greffé anhydride maléique. Deux voies ont été étudiées : l'irradiation gamma et l'oxydation par permanganate de potassium.

\section{Irradiation gamma}

\section{Principe}

L'oxydation par irradiation gamma consiste à exposer la poudrette à une source de Cobalt 60 afin de créer des fonctions oxydantes à sa surface. Les rayons gamma sont très pénétrants (ce qui permet un traitement industriel par sac, voire par palette) et le débit de dose est faible (2 kGy/h). La dose maximale est de 100 kGy; Au-delà, le traitement n'est plus industriellement rentable. La poudrette a ensuite été incorporée (taux de charge $30 \%$ ) dans une matrice PEhd/PEgMA. Deux méthodes ont été utilisées pour vérifier l'oxydation des poudrettes : la mouillabilimétrie et la spectroscopie infrarouge (mode ATR).

\section{Mouillabilité}

Le principe de cette méthode a été présenté au paragraphe 2-2. Les résultats sont représentés sur la figure 9.

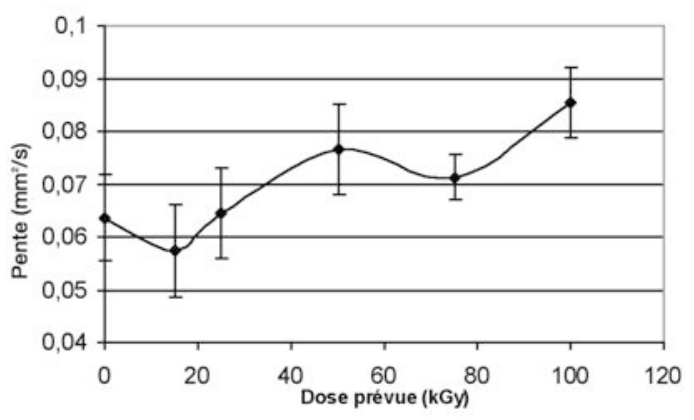

Figure 9 - Evolution de la vitesse d'absorption de l'éthand parla poudre Gen fonction de la dose dirradiation

L'augmentation de la vitesse d'absorption avec le taux d'irradiation indique que l'affinité entre la poudre et l'éthanol augmente. L'éthanol étant un solvant polaire, cela signifie que la poudre a été effectivement oxydée.

\section{Spectroscopie infrarouge}

La spectroscopie infrarouge a été utilisée ici pour observer la formation de nouvelles fonctions polaires. Le mode ATR est indispensable pour compenser l'absorption des ondes par le noir de carbone présent dans la poudre en quantité importante. Le graphe 10 présente les spectres obtenus dans la zone 1400 $2000 \mathrm{~cm}^{-1}$. On observe que le pic situé à $1636 \mathrm{~cm}^{-1}$, représentatif d'une fonction carbonyle augmente avec la dose reçue par la poudrette. Ce résultat concorde avec le précédent, à savoir que la poudrette a bien été oxydée par l'irradiation gamma. 


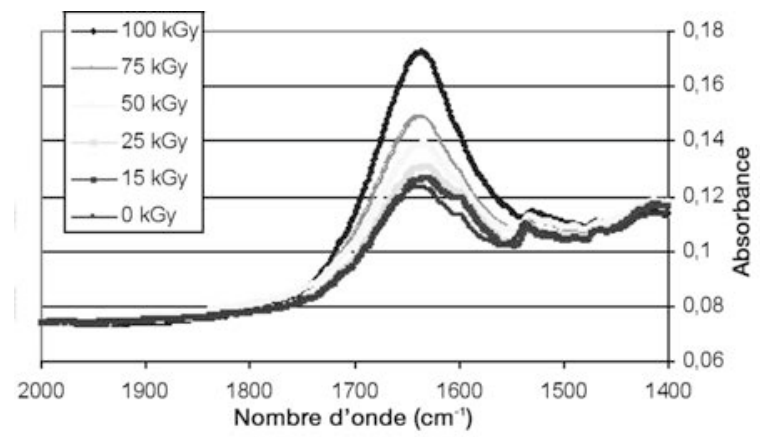

Figure 10 - Spectres infrarouges des poudres Girradiées entre 1400 et $2000 \mathrm{~cm}^{\prime \prime}$.

\section{Propriétés mécaniques}

Les performances mécaniques des mélanges réalisés avec les poudres irradiées sont présentées sur la figure I I. Bien que la surface des poudres ait été oxydée, aucune amélioration des propriétés mécaniques n'est perceptible. Ceci signifie que les groupes fonctionnels créés en surface des particules par irradiation ne créent pas de liaisons avec les fonctions anhydride du PEgMA ou sont en trop petite quantité.

Cette voie de compatibilisation ne semble donc pas être efficace.
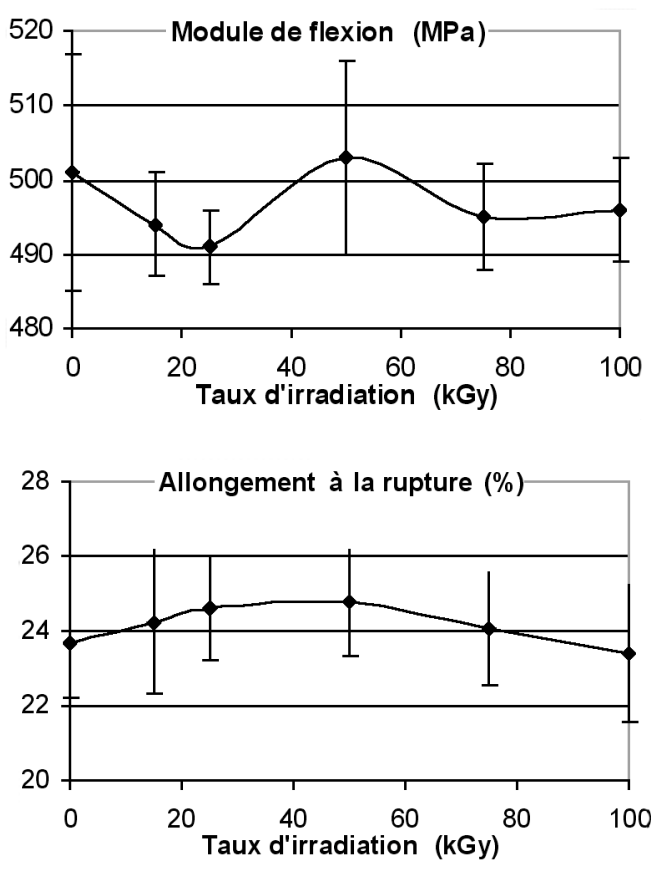

Figure 1 | - Propriétés mécaniques des mélanges PEhd/PEgMA/poudre G 65/5/30 en fonction du taux d'irradiation.

\section{Oxydation par permanganate de potassium}

\section{Principe}

Ce traitement reprend et poursuit celui proposé par Liu dans le cas de mélanges PP/poudre de caoutchouc [9]. L'auteur a proposé d'améliorer l'adhésion entre la matrice et la poudre en oxydant celle-ci par du permanganate de potassium et en l'incorporant dans une matrice contenant une petite quantité de PPgMA. La réaction supposée est la suivante:

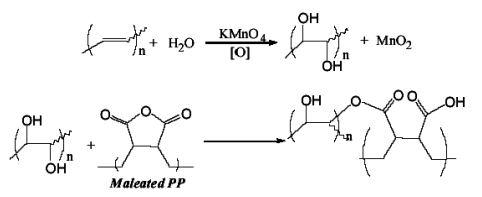

Figure 12 - Réaction présumée entre les fonctions $\mathrm{OH}$ en surface des particules et l'anhydride maléique du PEgMA [9].

La procédure utilisée par Liu est la suivante : la poudre est dispersée dans une solution eau/éthanol 90/10 contenant $2 \%$ de permanganate de potassium (en poids de poudre). Après 24 heures, la solution est lavée par une solution contenant du peroxyde d'hydrogène $\mathrm{H}_{2} \mathrm{O}_{2}$ (30 \%) et de l'acide sulfurique $(0,1 \%)$, pour éliminer le $\mathrm{MnO}_{2}$ adsorbé, puis par de l'eau déminéralisée. La poudre est finalement incorporée dans la matrice PP/PPgMA. Une amélioration des propriétés mécaniques (contrainte max et allongement à la rupture) est ainsi obtenue.

Dans notre cas, la même procédure a été appliquée avec un temps de traitement plus court ( $4 \mathrm{~h}$; nous avons considéré que la réaction était terminée d'après le changement de coloration de la solution) et plusieurs taux de $\mathrm{KMnO}_{4}$ (2, 10 et $20 \%$ ). Cependant, afin de vérifier la nécessité du lavage par $\mathrm{H}_{2} \mathrm{O}_{2}$, cette étape a été éliminée dans certains cas. La poudrette a ensuite été incorporée (taux de charge $30 \%$ ) dans une matrice PEhd/PEgMA (5 \% de PEgMA en poids du mélange) ou PEhd seul.

\section{Propriétés mécaniques}

Nous donnons ci-dessous les résultats des mesures du module de flexion obtenus pour les différents cas cités ci-dessus (figure 13).

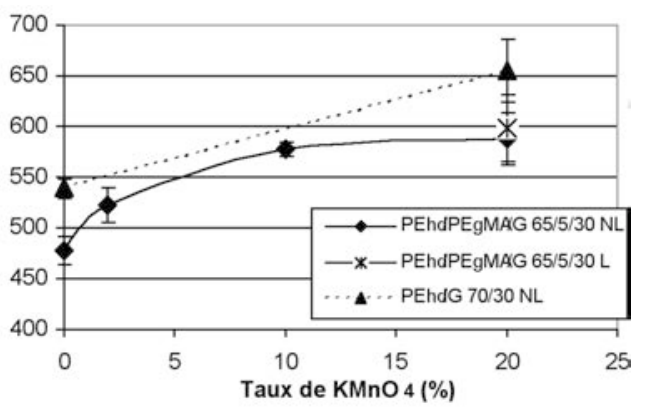

Figure 13 - Module de flexion (MPa) des mélanges PEhd/poudre G 70/30 et PEhd/PEgMA poudre G65/5/30 avec différents traitements de la poudre ( $L$ : lavage par $\mathrm{H}_{2} \mathrm{O}_{2}-\mathrm{NL}$ : Pas de lavage)

Les résultats montrent que le traitement par $\mathrm{KMnO}_{4}$ est efficace : le module évolue de 477 à $588 \mathrm{MPa}$ pour les mélanges contenant du PEgMA (poudre non lavée) lorsque le taux de $\mathrm{KMnO}_{4}$ augmente de 0 à $20 \%$, soit une augmentation de plus de $20 \%$. Cependant, on note que le lavage par peroxyde d'hydrogène est inutile : les mélanges PEhd/PEgMA/poudre oxydée $\left(20 \%\right.$ de $\mathrm{KMnO}_{4}$ ) présentent des modules identiques (598 et $588 \mathrm{MPa}$ ), que la poudre soit lavée ou non. 
On remarque aussi que le traitement est efficace sans utilisation de PEgMA. L'amélioration apportée par le traitement avec $20 \%$ de $\mathrm{KMnO}_{4}$ est dans ce cas de près de 22 \% (656 contre 539 $\mathrm{MPa})$. Le phénomène mis en jeu par ce traitement n'est donc pas celui proposé par Liu (figure 12).

Enfin, dans le cas des mélanges PEhd/PEgMAVG non lavée, au-delà de $10 \%$ de $\mathrm{KMnO}_{4}$, l'amélioration du module est plus marginale : le module est de $578 \mathrm{MPa}$ avec $10 \%$ de $\mathrm{KMnO}_{4}$ et $588 \mathrm{MPa}$ avec $20 \%$

Le traitement reste à optimiser et les phénomènes mis en jeu doivent être élucidés. Des essais complémentaires sont en cours pour comprendre ceux-ci. Nous pensons que la présence, en surface des particules, même après lavage par peroxyde d'hydrogène, de résidus à base de manganèse est responsable de l'amélioration des propriétés observée.

Cependant, il semble qu'un tel traitement (sans utilisation de PEgMA et surtout sans lavage par peroxyde d'hydrogène) soit efficace et envisageable d'un point de vue industriel.

\section{CONCLUSION}

Les travaux ci-dessus ont montré que l'incorporation de poudre de pneus dans une matrice polyoléfine peut être une voie intéressante de valorisation, pour autant que la chute des propriétés mécaniques consécutive à cette incorporation soit limitée. Dans le cadre de cette étude, nous pouvons dire que la granulométrie a une faible influence sur l'évolution de ces propriétés. Nous avons vu également que l'enrichissement en fibres par tamisage permet d'améliorer le module mais ne représente qu'une fraction minime des poudrettes.

Aussi est-il nécessaire d'envisager des traitements de surface pour améliorer l'affinité entre les particules et la matrice. L'oxydation des poudres par irradiation gamma, vérifiée par spectroscopie infrarouge et mouillabilimétrie, suivie de son incorporation dans une matrice contenant des groupes anhydride maléique, n'apporte aucune modification des propriétés mécaniques.

L'utilisation de permanganate de potassium, initialement pour oxyder la poudre et l'incorporer dans une matrice PEhd/PEgMA, a donné de bons résultats, bien que les mécanismes mis en jeu ne soient pas encore connus. La réaction proposée par Liu mettant en cause l'anhydride maléique n'est pas responsable de l'amélioration du module. De plus, le lavage par peroxyde d'hydrogène qui demande une grande quantité d'eau oxygénée et est fortement exothermique n'est pas nécessaire : Le traitement est dès lors applicable à une échelle industrielle.

\section{Références}

[1] Sheirs J., Polymer Recycling, 12, Ed. Wiley (1998).

[2] Adhikari B., De D., Maiti S., Prog Polym. Sci., 25, 909 (2000).

[3] Liu H.S., Mead J., Stacer R., Chelsea Center for Recycling and Economic Development, Technical Report 36 (200I).
[4] Ishiaku U.S., Chong C.S., Ismail H., PolymerTesting, I8, 62 I 633 (1999).

[5] Romero-Sanchez M.D., Pastor-Blas M.M., Martin-Martinez J.M., International Journal of Adhesion \& Adhesives 23, 49-57 (2003).

[6] Jong-kyu Park, Won-Tae Ju, Kwang-Hyun Paek, Yong-Hwan Kim, Yoon-Ho Choi, Ji-Hun Kim, Yong-Seok Hwang, Surface and Coatings Technology 174- |75, 547-552 (2003).

[7] Tyczkowski, I. Krawczyk, Wozniak B. Surface and Coatings Technology |74- |75, 849-853 (2003).

[8] Romero-Sanchez M.D., Pastor-Blas M.M., Martin-Martinez J.M., International Journal of Adhesion \& Adhesives 21, 325337 (200 I).

[9] Liu H.S., Richard C.P., Mead J., Stacer R., Chelsea Center for Recycling and Economic Development, Technical Report 18 (2000).

[10] Radhesh Kumar C., Fuhrmann I., Karger-Kocsis J., Polymer Degradation and Stability, 76, 137 (2002). 